Evaluation of the Xpert methicillin-resistant Staphylococcus aureus (MRSA) assay using the GeneXpert real-time PCR platform for rapid detection of MRSA from screening specimens. J Clin $\mathrm{Mi}$ crobiol 2008;46(10):3285-3290.

5. Senn L, Basset P, Nahimana I, Zanetti G, Blanc DS. Which anatomical sites should be sampled for screening of methicillinresistant Staphylococcus aureus carriage by culture or by rapid PCR test? Clin Microbiol Infect 2012;18(2):E31-E33.

\section{Multiple-Locus Variable Number Tandem Repeat Analysis Typing of Vancomycin- Resistant Enterococcus faecium in Serbia}

To the Editor-Multidrug-resistant Enterococcus faecium has become one of the most important nosocomial pathogens causing increasing numbers of nosocomial infections worldwide. In Serbia, after the first report of vancomycin-resistant Enterococci (VRE) in 2002, ${ }^{1}$ reports on the epidemiology of this bacterium have been scarce. ${ }^{2}$ Although pulsed-field gel electrophoresis (PFGE) was long considered the gold standard for typing methods, it has now been replaced by multilocus sequence typing (MLST). Molecular epidemiological studies using MLST identified host-specific genogroups, including 3 hospital-associated E. faecium lineages designated lineages 17, 18 , and $78 .{ }^{3}$ In order to obtain more insight into the molecular epidemiology of VRE in Serbia, we used the relatively fast and cheap multiple-locus variable number tandem repeat analysis (MLVA) typing method ${ }^{4}$ to determine the genetic relatedness of a total of $32 \mathrm{VRE}$ isolates isolated between 2007 and 2010 in the following hospitals: Clinical Center of Serbia (23), University Children's Clinic (2), and a gynecology clinic (1), all located in Belgrade, and 3 general hospitals located in Valjevo (8), Zaječar (2), and Cuprija (1; Table 1). In addition, we included 5 VRE isolates, each representative of the 5 major clones (based on PFGE, comprising 97 strains) of the 194 VRE isolated from hospitalized patients at 4 health institutions in Belgrade between 2002 and 2006 (data not shown).

The identification and antimicrobial susceptibility was performed by a Vitek system using IDGP and AST 586 card. All VRE were resistant to ampicillin, ciprofloxacin, streptomycin high doses, and imipenem and sensitive to linezolid. In total, $86.5 \%(32 / 37)$ of the strains were resistant to teicoplanin. Overall resistance to tetracycline was $70.3 \%(26 / 37)$, and overall resistance to gentamicin high doses was $75.7 \%$ (28/ 37).

MLVA typing of 37 VRE revealed 11 different MLVA types (MTs), including 4 not previously detected MTs (MT-340 to MT-343; Table 1). The most predominant MTs included MT$159(8 / 37$ [21.6\%]), MT-1 and MT-231 (5/37 [13.5\%] for both), MT-268 and MT-340 (4/37 [10.8\%] for both), and MT-296 and MT-342 (3/37 [8.1\%] for both). Unique new types were confined to patients residing at the Clinical Center of Serbia in Belgrade, while the other new types originated from inpatients in Belgrade and Valjevo. Except for MT-334, all MTs found outside Belgrade (MT-1 [3], MT-159 [2], MT231 [2], MT-296 [1], MT-340 [1], MT-342 [1]) were found in Belgrade as well, indicating widespread distribution of several types.

The most prevalent MT in this study, MT-159, is one of the most common types causing hospital outbreaks and invasive infections in Europe today. ${ }^{5-8}$ However, MT-159 was not identified among the 5 representative isolates of major clones of the 194 VRE, suggesting that MT-159 has spread recently and quickly, covering the territory of at least 2 metropolitan areas in Serbia. Only 2/8 members of that type were sensitive to high dozes of gentamicin, while resistance to all other antibiotics was uniform, suggesting possible clonal spread of that MT. Similar findings could be observed with MT-231, with $4 / 5$ members sensitive to tetracycline and resistant to all other antibiotics tested except linezolid.

In contrast, the second-most predominant type, MT-1, identified among $2 / 5$ representative isolates of major clones from Belgrade (representing 45/194 isolates) and in 2 other metropolitan areas of Serbia ( 3 strains), was the most widespread type in terms of time and space. Furthermore, that type was previously also identified among 2 VRE strains isolated at another hospital in Belgrade in $2005 .^{2}$ Some other studies that followed population changes of enterococci over long periods identified MT-1 from the beginning of the investigation and documented its span over 10 and 11 years, respectively. ${ }^{79}$

MLST analysis was performed on 12 isolates, including 1 representative of each MT that comprised more than 2 isolates, ${ }^{10}$ those with unique new $\mathrm{MT}$, and the 5 representatives of major clones from Belgrade and revealed in total 9 different sequence types (STs).

MT-1 is composed of multiple STs, thus making a polyclonal population. ${ }^{9}$ Indeed, 2 members of the MT- 1 type from our study have been ascribed different sequence types, ST17 and ST-554. Werner speculated that if isolates of ST-17 represent a rather ancient clonal type prone to recombination and thus divergent PFGE patterns for a long time, then ST203 and ST- 18 could constitute rather recent hospital-adapted clones. ST-203 from our study, ascribed to newly appearing MT-159, and newly introduced MLVA type MT-341 belonging to ST-18 support this hypothesis. Werner's speculation was based on German experience with molecular epidemiology of enterococci starting in the 1990s. It seems that evolution of VRE in Serbia could have a similar pattern. Hospital origin of at least 5 STs from our study (ST-17, ST-18, ST-252, ST426 , and ST-427), belonging to the Bayesian analysis of population structure subgroup 3-3, could be confirmed, according to Willems' findings. ${ }^{3}$

MLST proved its superiority over MLVA when 2 separate sequence types (ST-17 and ST-554), marked as 2 different pulsotypes by PFGE in 2 strains that could be distinguished, 
TABLE 1. Epidemiological and Molecular Typing Data on Vancomycin-Resistant Enterococcus faecium Recovered from 4 Metropolitan Areas in Serbia

\begin{tabular}{|c|c|c|c|c|c|c|c|c|c|c|c|c|c|c|c|c|}
\hline & Year & Source remarks & $\begin{array}{l}\text { Isolation } \\
\text { site }\end{array}$ & $\begin{array}{l}\text { MLVA } \\
\text { profile }\end{array}$ & MT & atpA & $\mathrm{ddl}$ & gdh & purK & gyd & pstS & adk & ST & $\mathrm{gm}$ & tet & tec \\
\hline $1^{\mathrm{a}}$ & 2004 & CITD & Urine & $5-7-3-3-2-3$ & 1 & 1 & 1 & 44 & 1 & 1 & 1 & 1 & 554 & $S$ & $\mathbf{R}$ & $\mathrm{R}$ \\
\hline $3^{a}$ & 2005 & Hematology & Urine & $5-7-1-4-2-3$ & 231 & 1 & 5 & 1 & 1 & 1 & 1 & 1 & 252 & $\mathrm{R}$ & $\mathrm{R}$ & $\mathrm{R}$ \\
\hline $4^{a}$ & 2005 & Hematology & Urine & $7-7-3-4-2-3$ & 268 & 7 & 5 & 1 & 18 & 1 & 1 & 1 & 427 & $\mathrm{~S}$ & $\mathbf{R}$ & $\mathrm{R}$ \\
\hline $5^{a}$ & 2006 & Hematology & Urine & $5-7-1-3-2-2$ & 296 & 1 & 3 & 1 & 1 & 1 & 7 & 1 & 552 & $\mathbf{R}$ & $\mathrm{R}$ & $\mathrm{R}$ \\
\hline 8 & 2007 & Hematology & Blood & $5-7-1-3-2-3$ & 340 & & & & & & & & & $\mathrm{R}$ & $\mathrm{S}$ & $\mathrm{R}$ \\
\hline 9 & 2007 & Children & Blood & $5-7-3-3-2-2$ & 7 & & & & & & & & & $\mathrm{R}$ & $\mathrm{R}$ & $\mathrm{R}$ \\
\hline 10 & 2007 & Children & CSF & $5-7-1-3-2-2$ & 296 & & & & & & & & & $\mathrm{R}$ & $S$ & $\mathrm{R}$ \\
\hline 11 & 2007 & Hematology & Blood & $5-7-3-3-2-2$ & 7 & & & & & & & & & $\mathrm{R}$ & $\mathrm{R}$ & $\mathbf{R}$ \\
\hline 12 & 2007 & ICU B & Blood & $7-7-3-4-2-3$ & 268 & & & & & & & & & $\mathbf{R}$ & $\mathrm{R}$ & $\mathrm{R}$ \\
\hline 17 & 2008 & Hematology & Urine & $5-7-3-3-1-2$ & 159 & & & & & & & & & $\mathbf{R}$ & $\mathbf{R}$ & $\mathrm{R}$ \\
\hline 18 & 2008 & Hematology & Urine & $5-7-3-3-1-2$ & 159 & & & & & & & & & $S$ & $\mathrm{R}$ & $\mathbf{R}$ \\
\hline 19 & 2008 & CITD & Urine & $5-7-3-3-1-2$ & 159 & 15 & 1 & 1 & 1 & 1 & 20 & 1 & 203 & $\mathrm{R}$ & $\mathrm{R}$ & $\mathrm{R}$ \\
\hline 20 & 2008 & CITD & Urine & $5-7-1-4-2-3$ & 231 & 1 & 5 & 1 & 1 & 1 & 1 & 1 & 252 & $\mathbf{R}$ & $S$ & $\mathrm{R}$ \\
\hline 21 & 2008 & CITD & Wound & $7-7-1-3-2-3$ & 342 & 7 & 5 & 1 & 18 & 1 & 1 & 1 & 427 & $\mathbf{R}$ & $\mathbf{R}$ & $\mathbf{R}$ \\
\hline 22 & 2008 & $\mathrm{TBC}$ & Urine & $5-7-1-4-2-3$ & 231 & & & & & & & & & $\mathrm{R}$ & $S$ & $\mathrm{R}$ \\
\hline 23 & 2009 & Valjevo & Wound & $5-7-3-3-2-3$ & 1 & & & & & & & & & $S$ & $S$ & $\mathrm{R}$ \\
\hline 24 & 2009 & Valjevo & Blood & $5-7-3-3-1-2$ & 159 & & & & & & & & & $\mathrm{R}$ & $\mathrm{R}$ & $\mathrm{R}$ \\
\hline 25 & 2009 & Valjevo & Urine & $5-7-3-3-1-2$ & 159 & & & & & & & & & $\mathbf{R}$ & $\mathrm{R}$ & $\mathrm{R}$ \\
\hline 26 & 2009 & Valjevo & Abscess & $5-7-1-4-2-3$ & 231 & & & & & & & & & $\mathrm{R}$ & $S$ & $S$ \\
\hline 27 & 2009 & Hematology & Blood & $5-7-1-3-2-3$ & 340 & 1 & 3 & 1 & 1 & 1 & 7 & 1 & 552 & $\mathrm{R}$ & $S$ & $\mathbf{R}$ \\
\hline 35 & 2010 & Valjevo & Blood & $5-7-1-4-2-3$ & 231 & & & & & & & & & $\mathrm{R}$ & $\mathrm{S}$ & $\mathrm{R}$ \\
\hline 36 & 2010 & Hematology & Wound & $5-7-1-3-2-3$ & 340 & & & & & & & & & $S$ & $\mathrm{R}$ & $\mathrm{R}$ \\
\hline 37 & 2010 & Hematology & CVC & $5-7-3-2-2-2$ & 341 & 7 & 1 & 1 & 1 & 5 & 1 & 1 & 18 & $\mathbf{R}$ & $\mathrm{R}$ & $S$ \\
\hline
\end{tabular}

Nоте. All vancomycin-resistant Enterococci were resistant to ampicillin, ciprofloxacin, streptomycin high doses, and imipenem and sensitive to linezolid. Children, Children's Hospital in Belgrade; Gynecology, Gynecology Clinic in Belgrade; CITD, Clinic for Infectious and Tropical Diseases of Clinical Center of Serbia; Hematology, Hematology Clinic of Clinical Center of Serbia; ICU B, Intensive Care Unit B of Clinical Center of Serbia; TBC, Clinic for Pulmonary Diseases of Clinical Center of Serbia; Valjevo, Zaječar, Cuprija: general hospitals covering metropolitan areas; CSF, cerebrospinal fluid; CVC, central venous catheter; MLVA, multiple-locus variable number tandem repeat analysis; gm, gentamicin; tet, tetracycline; tec, teicoplanin.

a Strains from the previous study.

were both assigned as MT-1. But this study also documented the opposite: 2 different MTs (MT-296 and MT-340) could be distinguished belonging to the same ST-552, and another pair, MT-268 and MT-342, shared the same ST-427. In conclusion, together with other typing techniques, MLVA was a useful tool that helped us to gain insight into the life and evolution of hospital-associated E. faecium in our country.

\section{ACKNOWLEDGMENTS}

Financial support. This article was supported by a Federation of European Biochemical Societies travel grant for 2009 and a United Nations Educational, Scientific, and Cultural Organization-American Society for Microbiology travel award for 2006 to perform a study of the collection of vancomycinresistant Enterococci at the microbiology laboratory of the Clinic for Infectious and Tropical Diseases, Clinical Center of Serbia, Belgrade. The study was 
performed at the research laboratory (Director, Marcus Zervos, MD, PhD) of Henry Ford Hospital in Detroit, Michigan, and the research laboratory of Medical Center Utrecht (Director, Rob Willems, MD, $\mathrm{PhD}$ ).

Potential conflicts of interest. All authors report no conflicts of interest relevant to this article. All authors submitted the ICMJE Form for Disclosure of Potential Conflicts of Interest, and the conflicts that the editors consider relevant to this article are disclosed here.

\section{Milica Jovanović, $\mathrm{MD}, \mathrm{PhD}{ }^{1}$ Janetta Top, $\mathrm{PhD}{ }^{2}$ Eline Majoor, Bsc; ${ }^{2}$ Marcus Zervos, $\mathrm{MD}, \mathrm{PhD} ;{ }^{3,4}$ Tanja Tošić, MD; ${ }^{1}$ Branka Stošović, $\mathrm{MD} ;{ }^{1}$ Lidija Lavadinović, $\mathbf{M D}{ }^{5}$ Rob Willems, $\mathbf{P h D}^{2}$}

Affiliations: 1. Department of Microbiology, Clinical Center of Serbia, Belgrade, Serbia; 2. Department of Medical Microbiology, University Medical Center Utrecht, Utrecht, The Netherlands; 3. Henry Ford Health System, Detroit, Michigan; 4. Wayne State University School of Medicine, Detroit, Michigan; 5. Clinic for Infectious and Tropical Diseases, Clinical Center of Serbia, Belgrade, Serbia.

Address correspondence to Milica Jovanović, $\mathrm{MD}, \mathrm{PhD}$, Bulevar Oslobodjenja 16, Belgrade 11000, Serbia (mijovan@eunet.rs).

Infect Control Hosp Epidemiol 2013;34(12):1337-1339

(C) 2013 by The Society for Healthcare Epidemiology of America. All rights reserved. 0899-823X/2013/3412-0024\$15.00. DOI: $10.1086 / 673990$

\section{REFERENCES}

1. Stošović B, Stepanović S, Donabedian S, et al. Vancomycinresistant Enterococcus faecalis in Serbia. Emerg Infect Dis 2004; 10:157-158.

2. Libisch B, Lepšanović Z, Top J, et al. Molecular characterization of vancomycin-resistant Enterococcus spp. clinical isolates from Hungary and Serbia. Scand J Infect Dis 2008;40:778-784.

3. Willems RJ, Top J, van Shalk W, Leavis $H$, et al. Restricted gene flow among hospital subpopulations of Enterococcus faecium. MBio 2012;3:e00151-12.

4. Top J, Schouls LM, Bonten JM, Willems RJL. Multiple-locus variable-number tandem repeat analysis, a novel typing scheme to study the genetic relatedness and epidemiology of Enterococcus faecium isolates. J Clin Microbiol 2004;42:4503-4511.

5. Billström H, Top J, Edlund C, et al. Frequent occurrence of multidrug-resistant $\mathrm{CC17}$ Enterococcus faecium among clinical isolates in Sweden. J Appl Microbiol 2010;108:1810-1816.

6. Weisser M, Oostdijk EA, Willems RJL, et al. Dynamics of ampicillin-resistant Enterococcus faecium clones colonizing hospitalized patients: data from a prospective observational study. BMC Infect Dis 2012;12:68.

7. Top J, Willems R), van der Velden S, et al. Emergence of ampicillin resistant CC17 Enterococcus faecium (AREfm) in the Netherlands. J Clin Microbiol 2008;46:214-219.

8. Borgmann S, Schulte B, Wolz C, et al. Discrimination between epidemic and non-epidemic glycopeptide-resistant $E$. faecium in a post-outbreak situation. J Hosp Infect 2007;67:49-55.

9. Werner G, Klare I, Witte W. The current MLVA typing scheme for Enterococcus faecium is less discriminatory than MLST and PFGE for epidemic-virulent, hospital-adapted clonal types. $B M C$ Microbiol 2007;7:28.

10. Homan WD, Tribe S, Poznanski M, et al. Multilocus sequence typing scheme for Enterococcus faecium. J Clin Microbiol 2002; 40:1963-1971.

\section{Clostridium difficile 027 Emerging Outbreak in Marseille, France}

To the Editor-Toxigenic Clostridium difficile cause pseudomembranous colitis that may be fatal. ${ }^{1}$ Typically, symptomatic C. difficile infections are hospital associated and preferentially involve elderly patients (greater than 65 years of age) recently treated with antibiotics. ${ }^{1,2}$ The clinical manifestations range from moderate diarrhea to serious manifestations. In 2003, an especially virulent clone, the ribotype 027 , was described in North America and was associated with an increased risk of death. ${ }^{2}$ Different outbreaks have been described in the past few years in northern Europe, ${ }^{3}$ particularly in Scotland, Germany, Austria, the Netherlands, Belgium, and northern France..$^{3-6}$ In France, a large outbreak was described in the Nord-Pas de Calais region during 2006-2007 with a mortality rate close to $30 \%$; since then, small clusters have been reported in Picardie, Rhône-Alpes, Lorraine, and Ille et Vilaine ${ }^{6}$ but not in the south of France. Cases reported from southern Europe, such as from Italy or Spain, remain sporadic to date. $5,7,8$

Marseille is a large city with approximately 850,000 inhabitants, and 112,000 patients are hospitalized in public hospitals in Marseille each year. We developed the point-of-care laboratory, which allows the use of both molecular and immunodetection tests to facilitate decision-making regarding the management of infectious diseases and patient care. ${ }^{9}$ The Xpert $C$. difficile Epi polymerase chain reaction (PCR) assay was used from April 2012 in the 2 point-of-care laboratories located in the Timone hospital and the North hospital. ${ }^{9}$ The Xpert $C$. difficile Epi PCR assay is a multiplex real-time PCR that detects the toxin $B$ gene $(t c d B)$, the binary toxin gene $(c d t)$, and the $t c d C$ gene deletion at nt 117 , identifying the 027 ribotype. ${ }^{10}$

From March 18, 2012, to July 5, 2013, we tested 2,205 stool samples for $C$. difficile toxin gene and detected 92 positive samples $(4.2 \%)$ at the point-of-care laboratory. Of these, 10 were positive for $C$. difficile 027; these were, to the best of our knowledge, the first cases due to this strain in Marseille. The index case was community acquired and was diagnosed in March 2013; this was followed by a rapid increase in the number of cases within a few weeks. Among these cases, 4 patients had been hospitalized for at least 48 hours, and 5 other patients had been hospitalized in the 3 preceding months. Among the 10 patients, 6 were or had been hospitalized in the same long-term care facility (Fig. 1). Finally, 2 patients were within the same family, and the delay between the onset of symptoms ( 3 weeks) might suggest that the first patient transmitted the infection to her father (Fig. 1). Patients were isolated after the diagnosis, and to date, no secondary cases have been described in our hospital.

The patients were mainly female $(70 \%)$ and older (mean 